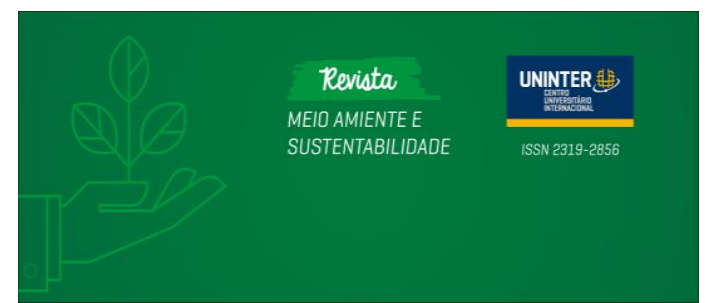

Revista Meio Ambiente e Sustentabilidade

Versão on-line ISSN 2319-2856

Volume 15, número 7. Curitiba - PR. jun/dez - 2018

\title{
Gestión de los residuos sólidos urbanos en el municipio de Quelimane - Mozambique
}

\author{
Felizardo Bernardo Camões \\ felizardocamoes@hotmail.com
}

Doutorando em Projeto pela Fundação Universitária Iberoamericana-FUNIBER de Moçambique. Docente na Universidade Pedagógica de Moçambique onde leciona as disciplinas Sociologia Ambiental e História do Pensamento Econômico.

Miguel Y. Ramírez-Sánchez miguelyramirez@hotmail.com

Graduado em Informática, mestre em Direção Estratégica, MBA em Business Administration e doutor em Educação. Pesquisador e docente da Universidad Internacional Iberoamericana do México.

Rodrigo Florencio da Silva rodriggo_florencio@hotmail.com

Doutorado em Meio ambiente e Desenvolvimento pelo Instituto Politécnico Nacional do México, mestre em Educação pela Universidad Interamericana de Desarrollo e licenciado em Letras Portuguesas e Ingles pela Universidade Guarulhos e em Letras Espanholas pela Universidade de Santo André.

\section{RESUMEN}

Esta investigación tiene como objetivo principal el análisis del proceso de gestión de residuos sólidos y surge de las observaciones realizadas en el municipio de Quelimane Mozambique. Se revisó la literatura científica sobre la temática, abordándose el trabajo en campo y generándose observaciones sobre el estado que guardan la gestión de los residuos sólidos urbanos hasta su destino final, por lo que se entrevistó la población seleccionada para este artículo para contar con un mejor enfoque sobre este aspecto en la investigación, obteniéndose resultados que impactan la gestión pública del municipio, ya que el nivel de atención a la gestión depende de la ubicación del hogar de los habitantes y se requiere urgentemente capacitación a los funcionarios y programas de sensibilización a la población.

Palabras-clave: Residuos sólidos urbanos, gestión de residuos; Quelimane Mozambique. 
Esta pesquisa tem como objetivo principal analisar o processo de gerenciamento dos resíduos sólidos e decorre das observações feitas no município de Quelimane - Moçambique. A literatura científica sobre o assunto foi revisada, abordando o trabalho no campo e gerando comentários sobre o estado da gestão dos resíduos sólidos urbanos até seu destino final, de modo que a população selecionada foi entrevistada, para que este artigo tenha um foco melhor neste aspecto na pesquisa, obtendo resultados que afetam a gestão pública do município, uma vez que o nível de atenção à gestão depende da localização do domicílio dos habitantes e o treinamento é urgente para funcionários e programas de conscientização para o população.

Palavras-chave: Resíduos sólidos urbanos, gestão de resíduos; Quelimane - Moçambique.

\section{INTRODUCCIÓN}

El municipio de Quelimane está localizado en la región centro de Mozambique en la provincia de Zambézia. De acuerdo con el documento del Instituto Nacional de Estadística de Mozambique - INE (2017), el número de habitantes del municipio en el censo de 2017 fue de 349.842 .

Quelimane es considerado el polo de desarrollo de la región y sus condiciones naturales son favorables para la plantación y el cultivo de palmas, de árboles de naranjas y limones como también de otras variedades frutas. Posee también un puerto fluvial e infraestructura adaptada a la navegación marítima de embarcaciones de gran porte.

La economía del municipio de Quelimane es dependiente del comercio, de la industria pesquera y del almacenamiento de los productos agrícolas como el arroz, frijoles, yuca, repollo y nuez de la india.

Tomando como punto de partida algunas debilidades actuales en la gestión y tratamiento de los residuos sólidos en el municipio de Quelimane, se sugieren alternativas para mejorar los procesos de recolección y eliminación de los residuos sólidos, así como también para la reducción de los gastos asociados a la enorme fuerza de trabajo de la empresa pública municipal de Quelimane responsable por ese servicio. 
Figura 1: Mapa de la región de Quelimane - Mozambique.

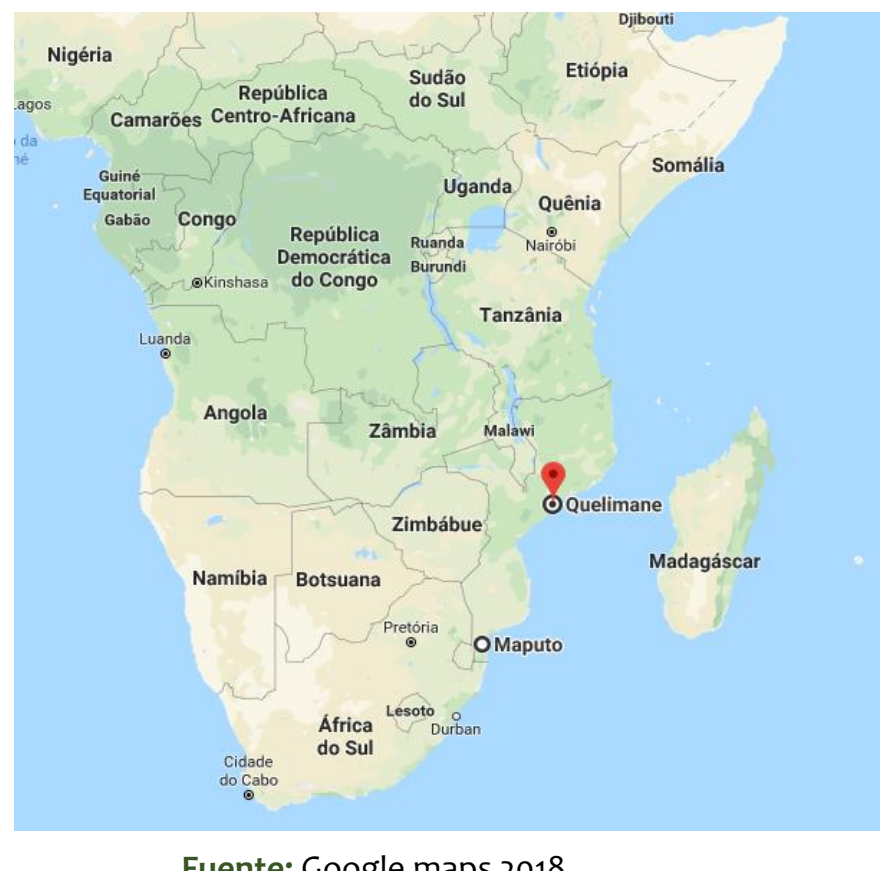

Fuente: Google maps 2018

Se parte del hecho de que los sistemas de limpieza urbana, juntamente con la ruptura de los ductos de los sistemas de suministro de agua, drenaje pluvial, que componen el saneamiento básico en Quelimane, representan un elevado interés para la salud pública y la conservación del medio ambiente, ya que garantiza la calidad de vida de los pobladores del municipio.

De acuerdo con la Publicação Oficial da República de Moçambique (2018), las autoridades municipales son las responsables por la gestión de la basura urbana. Aunque las autoridades tengan la incumbencia legal de garantizar los servicios de limpieza urbana basándose en el presupuesto legal, existe la necesidad de verificar si los recursos que el municipio dispone van en alcance de las necesidades para la prestación de estos servicios básicos prestados a los habitantes y a la promoción de salud, desarrollo local y municipio saludable.

Los ciudadanos generan los residuos sólidos en las residencias, comercios e industrias y partir de los productos que compran, condicionan los residuos que deben presentar en los puestos de entrega voluntaria para la colecta por medio de los camiones y tractores exclusivos para esta finalidad. 
Así, los sistemas de limpieza urbana para ser implementados, operados y mantenidos dentro de los patrones necesitan de los recursos financieros y de la participación de la comunidad.

\section{SITUACIÓN ACTUAL DE LA GESTIÓN DE LOS RESIDUOS SÓLIDOS URBANOS EN EL MUNICIPIO DE QUELIMANE}

El depósito de los residuos sólidos urbanos doméstico e industrial en Quelimane es realizado al aire libre por la inexistencia de un contenedor para su efecto. Con base en el documento del Conselho Municipal de Queliname (2016), esta problemática empezó a llegar a niveles extremos y alarmantes cuando se cerró el contenedor de basura a lo largo del camino de Quelimane Madal a principios de 1997, ya que con el éxodo rural, la población se fue aproximando a las ciudades por la búsqueda de mejores condiciones de vida y debido también a la guerra de los 16 años entre el Frente de Liberación de Mozambique (FRELIMO) y la Resistencia Nacional Mozambicana (RENAMO), contribuyendo extremadamente a la migración de personas por la búsqueda de lugares seguros.

Por consiguiente, las áreas que habían sido reservadas para el depósito de residuos sólidos, aunque sin condiciones para una gestión adecuada de los mismos, terminaron siendo ocupadas, generando nuevos problemas, principalmente por las casas próximas a los contenedores de basura, provocando una situación incómoda para esos habitantes, además de contaminar las salinas y los manglares alrededor, la proliferación de moscas, criaderos de mosquitos, olores nauseabundos, que empezaron a constituir un riesgo para la salud pública, principalmente a la zona donde era depositada la basura originando enfermedades endémicas como la malaria, diarrea y cólera.

Camões (2016) afirma que las condiciones climáticas constituyen factores importantes que pueden condicionar la descomposición de los residuos sólidos, consecuentemente la contaminación del suelo, de las aguas y la eclosión de enfermedades.

Actualmente, la remoción de los residuos sólidos urbanos en Quelimane es deficiente y su gestión es hecha de forma diferenciada, siendo que en los barrios donde las calles son asfaltadas y en el núcleo central de la ciudad, la colecta de basura 
es hecha diariamente a partir de los contenedores en los locales definidos. Ya en los mercados, la colecta es realizada en días alternados como en el mercado Brandão, Central y Aeroporto, y en los mercados de medio y pequeño porte que son dos veces en la semana.

El transporte de los residuos es realizado a través de tractores y depende de la disponibilidad y de las condiciones mecánicas.

Figura 2: Basurero de Quelimane - Mozambique.

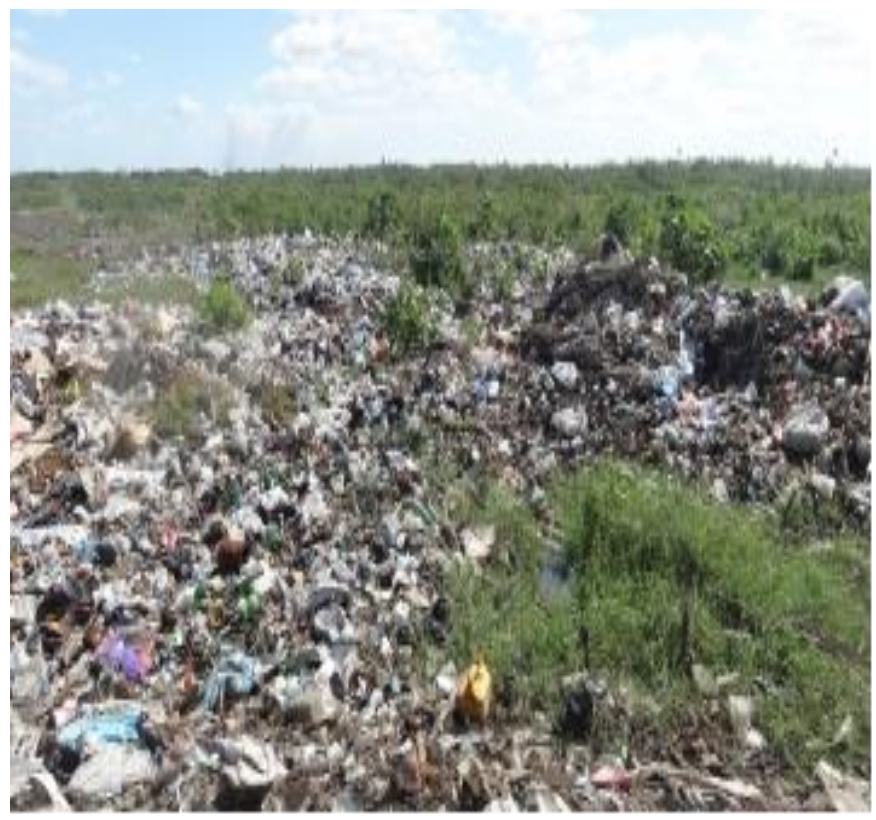

Fuente: Autores 2018

De acuerdo con el documento semestral de actividades de la Empresa Municipal de Saneamiento Ambiental - EMUSA - Moçambique (2016), el local elegido para el depósito de los residuos en Quelimane dista a $5 \mathrm{~km}$, a pesar de las precarias condiciones. Sin embargo, se nota que los residuos sólidos urbanos son abandonados sin ningún tratamiento previo, contaminando el agua subterránea de la zona.

Debido la falta de espacio y el conflicto político ideológico que se vive en Quelimane, la apertura de un atierro sanitario está distante de solucionar esta problemática. La ciudad de Quelimane tiene dos administraciones que en algunos casos generan conflictos entre ellas. Aunque la ciudad de Quelimane es administrativamente un municipio con un gobierno local electo y el distrito donde está insertado el municipio administrado por el gobierno central, en el cuadro del decreto de la ley 26/2013 de 18 de diciembre de 2013. Es importante mencionar que el 
municipio de Quelimane es gestionado por la oposición, hecho que propicia la generación de conflicto, dando espacios de maniobras y complicando la vida de los ciudadanos y sus derechos que no son respetados, generando un ambiente con pésimas condiciones de saneamiento.

Para evitar la proliferación de enfermedades, es necesario la integración de esfuerzos entre el Consejo Municipal y el Gobierno del Distrito de Quelimane para poder identificar un área que sirva para la implementación del futuro atierro sanitario.

Dos Muchangos, Tokay y Hanashima (2015) comentan que la gestión de los desechos se encuentra entre los problemas más complejos en las áreas urbanas y para el abordaje del tema, es necesario una planificación de los residuos y una forma de gestión para la problemática.

La plataforma VD Atlas International (2015) menciona que el Decreto 94/2014 refuerza la necesidad de que se defina el cuadro legal en que se deberá procesar la gestión de residuos en el territorio nacional.

Cuando la basura urbana es simplemente tirada al aire libre, por ejemplo, el local se torna en una fuente de emisión del metano $\left(\mathrm{CH}_{4}\right)$, gas resultante de la descomposición de la materia orgánica presente en los residuos sólidos urbanos. Silva y Campos (2008) afirman que el gas metano cuando es lanzado a la atmosfera terrestre es 21 veces más nocivo que el $\mathrm{CO}_{2}$, potencializando el calentamiento global.

El abandono de los residuos, principalmente los que están junto a los mercados y en los barrios periféricos beneficiados por el sistema de drenaje, obstruye el funcionamiento normal del sistema y complica aún más el proceso de gestión de los residuos sólidos.

Mbiba (2014) menciona que a pesar de que en la última década hubo una mayor conciencia y esfuerzo para implementar la gestión de los residuos sólidos en las ciudades africanas, este continúa siendo un problema crónico.

Los pocos contenedores que existen no son suficientes para la cobertura de toda la ciudad y la situación se agrava más con la población que no respeta la hora y los días de la entrega voluntaria de residuos, transbordando los contenedores e incinerando la basura en los propios contenedores causando daños en la estructura metálica de los mismos. 
Con campañas de sensibilización y de educación ambiental, los munícipes podrían ser concientizados acerca de los problemas generados al depositar la basura fuera de su día de colecta como también su incineración.

Jequecene, Sánchez y da Silva (2017, p.149) mencionan que:

La Educación Ambiental Surgió Con La Perspectiva De Garantizar La Participación Pública A Través De La Disponibilidad De Información Útil Para Que La Población Pudiera Entender Y Evaluar El Grado De Riegos Resultantes De Las Malas Prácticas Ambientales.

Se considera que es por falta de educación ambiental que los munícipes depositan y avientan los residuos en las vías públicas, dentro de sus propios patios y en las esquinas al revés de depositar en los locales adecuados.

Con lo anterior, Florencio da Silva (2017, p.244) aborda que:

Con la Educación Ambienta es posible desarrollar una nueva educación con propuesta que contribuya al cambio social y la solución de los problemas ambientales actuales, fomentando de forma inequívoca el respeto por toda forma de vida y cultura como respuestas diversas de adaptación y relacionamiento con el medio ambiente.

El depósito de basura en los manglares es otro factor que pone el peligro la existencia de la vida como también de las especies acuáticas allá existentes. La contaminación del agua, la situación sanitaria de las poblaciones que de esas especies dependen, el medio ambiente local y otros problemas asociados.

El principal problema focalizado en este estudio es la falta de saneamiento asociada al ineficiente sistema de gestión de los residuos sólidos.

Camões (2016) dice que se observa una deposición de residuos en las zarjas de drenaje y en los lugares al aire libre durante días sin que realice la retirada de esa basura, contribuyendo para que el municipio de Quelimane sea considerado una de las ciudades con mayor índice de ocurrencia de cólera y enfermedades diarreicas.

La gestión de los residuos sólidos en Quelimane presenta un cuadro con debilidad en cuanto la cobertura territorial del servicio de limpieza urbana ya que la colecta se concentra en ciertas regiones de fácil acceso, dejando la zona periurbana con discrepancia en el atendimiento de la colecta de la basura, aunque la población de esa zona pague los impuestos correspondientes.

Las autoridades tienen la obligación de responder las exigencias para lograr un desarrollo sustentable. A pesar de la existencia de muchas legislaciones, se verifica 
todavía una debilidad en los cumplimientos de las leyes. También la falta de recursos financieros y humanos amenaza el sistema de gestión local, comprometiendo la prestación de esos servicios esenciales.

Jacobi y Beses (2011, p.136) afirman que:

En ciudades de países en desarrollo con urbanización muy acelerada, se verifican déficits en la capacidad financiera y administrativa de esas en proporcionar infraestructura y servicios esenciales como el agua, saneamiento, colecta y destinación adecuada de la basura y vivienda, y en asegurar la seguridad y el control de la calidad ambiental para la población.

El poder municipal de Quelimane parece redimir sus responsabilidades sobre la gestión de los residuos urbanos a pesar de que la ley prevé que cualquiera que cometa infracción podrá pagar una multa. Ese pago es realizado en el momento de la compra de energía eléctrica a través de una tasa de basura y es legalizada por el Decreto 94/2014.

\section{METODOLOGÍA}

Para desarrollar este trabajo, se basó en la observación por parte de los investigadores que identificaron la ocurrencia de problemas diversos relacionados con la gestión de los residuos sólidos urbanos en el municipio de Quelimane debido la falta de gestión y/o la debilidad de la legislación para la sustentabilidad urbana a través de la promoción de la salud para el municipio saludable.

Esta investigación fue desarrollada en tres etapas, que se describen a continuación.

La primera se basó en la revisión de la literatura donde se determinó el "estado de arte" mostrándose a través de la literatura existente lo que ya fue investigado sobre la gestión de residuos sólidos, las brechas existentes del tema y esto permitió identificar los principales obstáculos teóricos y metodológicos, posibilitando el entendimiento y análisis del Reglamento de Gestión de Residuos Sólidos Urbanos (Decreto 94/2014, de 31 de diciembre de 2014) y del Reglamento de Gestión de Residuos Sólidos Peligrosos (Decreto 83/2014, de diciembre de 2014) en la 
medida en que fueran realizados análisis para evaluar la debilidad y la aplicación de estos documentos legales.

La revisión bibliográfica contribuyó para la obtención de información acerca de la situación actual del tema y problemática investigada, con la finalidad de insertar la investigación dentro de un cuadro de referencias teóricas para la explicación del porqué esta problemática es teórica y que no es generada o explicada por una teoría en particular. Aun en la etapa de revisión bibliográfica, se procedió a la revisión histórica, buscando recuperar la evolución de la gestión de los residuos y su manejo en el mundo y en el contexto local, en Mozambique y en Quelimane desde que se instituyó autoridades locales o poder local y /o órganos locales del Estado.

Abarcando acerca de la inserción de esa evolución dentro de un cuadro teórico de referencia, se explicó los factores determinantes y las implicaciones de los cambios. No obstante, se recorrió, en esta etapa, la consulta de literatura que abarca el proceso de gestión de residuos sólidos en Mozambique.

Para desarrollar la encuesta para los gestores municipales, se elaboró una lista sistematizadas de problemas relacionados a la gestión de residuos sólidos urbanos que fueron consultados en los diversos documentos institucionales como los guías de saneamiento, reportes e inventarios de residuos, procesos jurídicos y además de publicaciones científicas. Ya para los ciudadanos de Quelimane, se pretendió recolectar información acerca de la sensibilización de los servicios prestados por el municipio en la gestión de los residuos.

En la segunda etapa se desarrolló el trabajo de campo, donde se profundizó el objeto de estudio y se mantuvo contacto con las autoridades locales del municipio como los gestores, miembros de la asamblea municipal, secretarios de los barrios que están en estrecha conexión con las autoridades municipales y con los munícipes.

La gestión municipal de los residuos sólidos urbanos en Quelimane es realizada por la Empresa Municipal de Saneamiento (EMUSA), que es responsable por la limpieza urbana, colecta, transporte y disposición final de los residuos urbanos. Es función del Municipio asesorar el programa municipal de gestión de residuos y desarrollar campañas de educación y sensibilización ambiental junto a la población en el cuadro de la legislación de la gestión municipal. 
Los gestores municipales encuestados fueron entrevistados individualmente y todos ocupaban puestos de gerencia dentro de la estructura jerárquica del Municipio.

Antes de realizar las entrevistas y aplicar la encuesta, se aclaró el objetivo de la investigación. En esta etapa también se niveló la temática de sustentabilidad y su terminología y la lista de los problemas fue presentada a los entrevistados y se solicitó su percepción y que apuntaran los problemas categorizados en Quelimane y que identificaran otra problemática, en caso de no estar en la lista presentada.

La tercera etapa contempla el análisis los resultados e interpretación de las entrevistas, en donde fue seleccionado un muestreo intencional donde se eligió personas que trabajan directamente en el Consejo Municipal y los habitantes por ser ellos los afectados con la problemática de la gestión de residuos. Para eso, se seleccionó 117 individuos de ambos sexos y discriminados de la siguiente forma:

01 - Presidente del consejo Municipal / asesor;

04 - Miembros de la Asamblea Municipal;

01 - Jefe de los servicios urbanos del Consejo Municipal de Quelimane;

01 - Concejal del área de saneamiento del medio ambiente;

04 - Técnico de la EMUSA;

04 - Organizaciones no gubernamentales en que fue elegido un miembro de cada organización;

102 - Munícipes de la ciudad de Quelimane a ser elegidos 2 de cada 51 barrios existentes en la ciudad.

En la imposibilidad de obtener una representatividad de un 10\% de los elementos de cada barrio, se verificó que cerca de dos (2) elementos serian representativos para cada barrio, hecho que tornó el muestreo representativo. En este caso, el estudio contó con una muestra aproximadamente de 117 elementos.

\section{INSTRUMENTO DE INVESTIGACIÓN}

Se utilizaron los siguientes elementos para la realización de la recolección de los datos: 
- Guía de entrevistas dirigido a los munícipes

- Guía de encuesta dirigido a los órganos del Poder Público y a las ONG

- Ficha de observación que permitió observar en que locales son depositados los residuos sólidos, los horarios en que son recolectados y la frecuencia de esa actividad

- Las entrevistas fueron semi-estructuradas, adecuándose a los estudios cualitativos donde se buscó explotar las verbalizaciones incluyendo las de contenido afectivo.

De acuerdo con Zaneti (2003), una encuesta proporciona la posibilidad de que los sujetos de estudio manifiesten durante la entrevista sus creencias, valores, ampliando el cuadro de sus vivencias como individuos y miembros de un grupo.

Las entrevistas fueron direccionadas a los siguientes actores sociales:
a) Asesor del presidente del consejo Municipal;
b) Concejal del área de saneamiento del medio ambiente;
C) Técnicos de la empresa EMUSA;
d) Miembros de la Asamblea Municipal.

Los objetivos de las entrevistas visaban los siguientes aspectos por cada área:

a) Consejo municipal: evaluar el proceso de gestión de los residuos sólidos urbanos del municipio de Quelimane y verificar la perspectiva de las políticas públicas para el sistema de gestión de residuos

b) Asamblea Municipal: verificar la aplicabilidad de las leyes y la relación entre el legislador o ejecutivo y los munícipes de la ciudad.

c) Empresa Municipal de Saneamiento (EMUSA): verificar la concepción de la educación ambiental adoptada por la EMUSA para la movilización del cambio social de la actitud y del comportamiento, identificar los procedimientos educativos y técnicos de la gestión de los residuos sólidos urbanos y apuntar las restricciones que el sector de transporte enfrenta en la colecta de los residuos y el estado de la infraestructura en la visión de los técnicos. 
Para la selección de los entrevistados, se tomó en cuenta el conocimiento del área de estudio, el rango de edad superior a los 30 años y la profesión del encuestado.

\section{ANALISIS Y DISCUSION DE LOS RESULTADOS}

Para el análisis e interpretación de los datos, se aplicaron tres preguntas consideradas importantes en la gestión de los residuos urbanos que abarcaban la estructura organizacional del Consejo Municipal de Quelimane, los locales de depósito de los residuos sólidos urbanos en los puestos de entrega voluntario y la sustentabilidad económica y financiera para la prestación de los servicios básicos a los munícipes de la ciudad de Quelimane.

En la pregunta sobre la estructura del Consejo Municipal de Quelimane para la gestión de los residuos sólidos urbanos, se buscó entender la responsabilidad del saneamiento del medio en el Municipio. Esta cuestión fue respondida por el Poder Público, siendo que 2 de los 4 diputados municipales o concejales correspondientes, contestaron que en Quelimane existe una empresa creada dentro del Consejo Municipal que es la responsable por la gestión de los residuos sólidos mientras que, de los 4 miembros de la Asamblea Municipal, 3 reconocen la existencia de la empresa EMUSA, pero afirmaron que hay una mala prestación de servicio a la población. Se destaca también que un miembro de la Asamblea Municipal dijo no saber la existencia de una empresa responsable por la gestión de residuos sólidos y que, si hubiera una empresa, la ciudad no estaría llena de basura y no exhalaría olores desagradables.

Analizando la respuesta de los cuatro miembros de la Asamblea Municipal, se comenta que a pesar de haber divergencia sobre la estructura de la gestión de los residuos sólidos en Quelimane, ellos fueron unánimes al afirmar que el Municipio enfrenta muchos desafíos y los medios que la ciudad dispone no son los suficientes para dar soluciones a sus necesidades reales en el ámbito de saneamiento. Enfatizaron, adicionalmente que todo personal que labora en la EMUSA necesita de capacitación acorde a esta temática.

En relación a la existencia de depósito voluntario de residuos sólidos y el horario establecido para la entrega, el asesor del presidente del Consejo Municipal contestó que de manera positiva que el Consejo Municipal ha enviado esfuerzos para que la basura sea depositada en locales seguros y que el actual presidente del municipio 
luego que asumió Quelimane se preocupó en mandar a construir puestos de entrega voluntario de basura en las localidades que no habían contenedores, pero la situación de la ciudad debe ser analizada de forma holística y que los conciudadanos no están preparados para vivir en el medio urbano y muchos de ellos llegaron en el periodo armado y que vivían en los centros habitacionales y después de ese periodo, los que no regresaron a sus localidades de origen, se mantuvieron en la zona periférica y actualmente con la expansión de la ciudad, estas zonas hacen parte de la ciudad y los habitantes siguen con su mal hábito de tirar la basura de forma inconsciente.

El asesor del presidente del Consejo Municipal mencionó acerca del horario de entrega de los residuos que el Municipio estableció el periodo de 18-20h para la entrega de la basura, pero que el horario nunca era respetado por los munícipes. Agregó también que los ciudadanos tiraban la basura en el momento que el camión colector estaba a punto de realizar la colecta y que mucha gente del municipio aventaba la basura fuera del contenedor, dificultando el trabajo del personal encargado por la colecta.

En la tercera pregunta sobre la sustentabilidad económica y financiera para la prestación de los servicios básicos a los munícipes de Quelimane, los 8 miembros del Poder Público, 4 diputados municipales y 4 miembros de la Asamblea municipal fueron unánimes al decir que el Municipio recibe dinero del Gobierno Central, el llamado fondo de compensación autárquica. Además, mencionaron que la ciudad de Quelimane obtiene ingresos propios a través del pago de la tasa de basura que es cobrada junto con la electricidad de Mozambique en el momento de la compra de energía y del pago del consumo de agua.

De los 102 munícipes entrevistados sobre la tasa de la basura cobrada, 75 personas respondieron que esa tasa no satisface a los munícipes ya que hay momentos que la basura no es colectada, que los mendigos incineran los contenedores, que la colecta favorece las zonas asfaltadas y de fácil acceso y que al pagar la tasa de basura no existe un descuento para las zonas que son afectadas por la colecta. Ya los 27 ciudadanos restantes, hablaron de que hacen su parte y depositan la basura en los horarios y en los contenedores destinados, sin embargo, faltan medios humanos y materiales para cubrir toda la ciudad y que el Municipio debe ampliar esfuerzos para la satisfacción plena en la prestación de los servicios básicos a la población. 


\section{CONSIDERACIONES FINALES}

Se verificó que la gestión de residuos urbanos en Quelimane presenta un cuadro sombrío en lo que concierne a la cobertura territorial en la prestación de los servicios básicos de salubridad y limpieza urbana, colecta de los residuos sólidos a la zona asfaltada que es considerada la más privilegiada en relación con la zona periférica.

Esas cuestiones mencionadas se deben a la falta de mecanismos de una gestión eficaz y eficiente y de una estructura organizacional que responda las expectativas de los munícipes. Los puestos de entrega voluntaria construidos en los barrios no se adecuan a la realidad ya que existe una separación antes de su encaminamiento para los basureros.

Acerca del proceso de gestión de los residuos sólidos, este estudio constató que el morador de las zonas céntricas tiene más privilegio de colecta de los residuos mientras la mayoría entierra la basura dentro del patio de sus casas.

En el municipio de Quelimane no existe atierro sanitario y la basura es depositada al aire libre, generando peligros a la salud pública que, en periodo de lluvias, la ciudad sufre de la proliferación de enfermedades como la malaria, diarrea y cólera.

Además de los contenedores donde los ciudadanos depositan su basura, es muy común encontrar basura en los terrenos baldíos donde se encuentran animales que disputan el resto de alimentos con los mendigos.

Se concluye mencionando que para la gestión de los residuos sólidos en Quelimane es importante la creación de un equipo técnico multisectorial para apoyar al Consejo Municipal en la elaboración de planes de acción sobre la gestión de los residuos sólidos urbanos; la elaboración de un plan de gestión de los residuos sólidos urbanos; la revisión de la ley sobre las multas y castigo a los ciudadanos que no cumplen con la colecta correcta; la revisión sobre la tasa de la basura y un seguimiento del dinero recibido de esa tasa; la capacitación de los trabajados de la Empresa Municipal de Saneamiento Ambiental - EMUSA y la implementación de un sistema de monitoreo de la gestión de los residuos sólidos urbanos desde su colecta, tratamiento, transporte y destino final. 


\section{REFERENCIAS}

CAMÕES, F. Gestão de Resíduos Sólidos Urbanos no Município de Quelimane 20102015. In GSD et al. (Coord.). Reflexões sobre a Geografia Física e o Ambiente num mundo em conflito (Vol.1, Cap. 16, pp.178-185). Maputo: Editora Educar, 2016.

DOS MUCHANGOS, Leticia Sarmento; TOKAI, Akihiro; HANASHIMA, Atsuko. Analyzing the structure of barriers to municipal solid waste management policy planning in Maputo city, Mozambique. Environmental development, 2015, vol. 16, p. 76-89.

FLORENCIO DA SILVA, R. Sustentabilidade Educacional: A sensibilização do uso das energias renováveis através de uma estrategia educativa com alunos universitários. In C.B (Coord.). Educação \& Gestão: Modelos Educacionais e Métodos de Gestão Aplicados à Educação (Vol.1, Cap. 25, pp.243-250). Campinas: Lopes Editora, 2017.

JACOBI, Pedro Roberto; BESEN, Gina Rizpah. Gestão de resíduos sólidos em São Paulo: desafios da sustentabilidade. Estudos avançados, v. 25, n. 71, p. 135-158, 2011.

JEQUECENE, Domingos Brisito; SÁNCHEZ, Miguel Y. Ramírez; DA SILVA, Rodrigo Florencio. Impacto de la Educación Ambiental en las Comunidades Próximas del Parque Nacional de Gorongosa-Estudio de Caso Comunidad de Nhambita.. REMEARevista Eletrônica do Mestrado em Educação Ambiental, v. 34, n. 3, p. 147-165, 2017.

MBIBA, Beacon. Urban solid waste characteristics and household appetite for separation at source in Eastern and Southern Africa. Habitat International, 2014, vol. 43, p. $152-162$.

MOÇAMBIQUE. Conselho Municoal da Cidade de Quelimane. Relatório Anual de Actividades. Disponível

em: http://www.portaldogoverno.gov.mz/por/content/download/5565/39958/version/1/f ile/BR_46_III_SERIE_2016.pdf. Acesso em: 20 de fev. de 2018.

- Empresa Municipal de Saneamento Ambiental. Relatório Semestral de Actividades. Disponível em: http://www.portaldogoverno.gov.mz/por/content/download/5565/39958/version/1/f ile/BR_46_III_SERIE_2016.pdf. Acesso em: 27 de fev. de 2018.

Instituto Nacional de Estadística. Disponível em: http://www.ine.gov.mz/operacoes-estatisticas/censos/censo-2007/censo2017/divulgacao-os-resultados-preliminares-iv-rgph-2017. Acesso em: $01 \mathrm{de}$ mar. de 2018.

Publicação Oficial da República. Disponível em: http://www.portaldogoverno.gov.mz/por/content/download/3813/29186/version/1/fi le/BR+51+III+SERIE+SUPLEMENTO+3.pdf. Acesso em: 28 de jan. de 2018. 
SILVA, Tiago Nascimento; CAMPOS, Lucila Maria de Souza. Avaliação da produção e qualidade do gás de aterro para energia no aterro sanitário dos BandeirantesSP. Engenharia Sanitária e Ambiental, v. 13, n. 1, p. 88-96, 2008.

VDATLAS International Platform. Ambiente | Moçambique: Novos Regulamentos de Gestão de Resíduos. Disponível em: http://www.vda.pt/xms/files/Newsletters/2015/Flash_VdAtlas_Mocambique_-

Ambiente__Novos_Regulamentos_de_Gestao_de_Residuos_-16.05.2015-.pdf. Acesso em: 28 de fev. de 2018.

ZANETI, I.C.B. Educação ambiental, resíduos sólidos urbanos e sustentabilidade. Um estudo de caso sobre o sitema de gestão de Porto Alegre. 2003. 176 f. Dissertação (Doutordo em Política e Gestão Ambiental) -Centro de Desenvolvimento Sustentável, Universidade de Brasília, Brasília. 2003. 